\title{
On Lambe's infinite integral formula
}

\author{
By A. ERdélyu.
}

(Received 1st November, 1939. Read 25th November, 1939.)

In a recent paper (these Proceedings (2), 6 (1939), 75-8), C. G. Lambe established, and gave some applications of, the formula

$$
f(x+z)=\frac{1}{2 \pi i} \int_{-i \infty}^{i \infty} \Gamma(-s)(-z)^{s}\left\{D^{s} f(x)\right\} d s,
$$

in which $D^{s}$ is the symbol for the derivative of fractional order $s$. Lambe's proof of (1) is not quite rigorous and it does not bring out the conditions which have to be imposed upon $f(x)$ in order to make (1) true. Furthermore this proof does not give any evidence as to the definition of fractional derivative which is to be used in connection with (1).

Readers familiar with the theory of the Mellin Transform will presently realise that the opinion "For the purposes of this formula, any definition [of fractional derivatives] may be adopted which is consistent with the $N$-th differential coefficient when $s=N$, an integer," is not likely to be true. For (1) is but the Mellin-inversion (E. C. Titchmarsh, Introduction to the Theory of Fourier Integrals (1937), (1.5.1) and (1.5.2)) of

$$
\Gamma(-s) D^{s} f(x)=-\int_{0}^{\infty} f(x+z)(-z)^{-s-1} d z,
$$

so that it is rather plausible that (1) is only compatible with definition (2) of fractional derivatives.

Confining myself for the sake of simplicity to the case of real variables $x, y$, I shall prove that under certain conditions (1) and (2) are equivalent.

Let $\Gamma(-k-i u)\left\{(-D)^{k+i u} f(x)\right\}$ be a measurable function of the real variable $u$ and let the Lebesgue integral of this function over $(-\infty,+\infty)$ be absolutely convergent; suppose that this function is of bounded variation in the neighbourhood of the point $u=t$. Then from

$$
f(x+y)=\frac{1}{2 \pi i} \int_{k-i \infty}^{k+i \infty} \Gamma(-s) y^{8}\left\{(-D)^{s} f(x)\right\} d s
$$

follows :

(4) $\frac{1}{2}\left\{(-D)^{s+i 0} f(x)+(-D)^{s-i 0} f(x)\right\}=\frac{1}{\Gamma(-s)} \lim _{\lambda \rightarrow \infty} \int_{1 / \lambda}^{\lambda} f(x+y) y^{-\varepsilon-1} d y$,

$$
(s=k+i t) \text {. }
$$


The validity of this follows from Theorem 29 of Titchmarsh's Fourier Integrals. If $(-D)^{8} f(x)$ is a continuous function of $t,(s=k+i t)$, and if the integral on the right hand side converges, then we may write instead of (4)

or

$$
(-D)^{8} f(x)=\frac{1}{\Gamma(-s)} \int_{0}^{\infty} f(x+y) y^{-8-1} d y,
$$

$$
(-D)^{8} f(x)=\frac{1}{\Gamma(-s)} \int_{x}^{\infty}(t-x)^{-s-1} f(t) d t .
$$

Conversely it is easy to prove that the definition (5) of fractional derivatives involves Lambe's formula (3). For, due to Theorem 28 of Fourier Integrals, the following statement holds true:

Let $f(t)$ be a measurable function of the real variable $t$, of bounded variation in the neighbourhood of the point $t=x+y$; suppose the definition (5) of fractional derivatives to be absolutely convergent for some $\mathfrak{R}(s)=k$ in the sense of Lebesgue integrals. Then

(6) $\frac{1}{2}\{f(x+y+0)+f(x+y-0)\}=\frac{1}{2 \pi^{i}} \lim _{T \rightarrow \infty} \int_{k-i T}^{k+i T} \Gamma(-s) y^{8}\left\{(-D)^{8} f(x)\right\} d s$.

If $f(t)$ is continuous at the point $t=x+y$, and if the integral on the right hand side is convergent, then (6) may be replaced by (3).

Thus it is seen that Lambe's formula is exactly the inversion of definition (5) of fractional derivatives and can not be used, in general, in connection with any other definition of generalised differential coefficient.

The restrictions imposed in this note upon the functions dealt with are not the only ones possible. Using instead of the theorems quoted above the theory of Mellin Transforms of class $L^{p}$ (Fourier Integrals, Theorem 86), other conditions of validity could be obtained. The extension to complex variables is obvious (Cf. e.g. G. Doetsch, Theorie und Anwendung der Laplace Transformation (1937), p. 114 et. seq.).

The Mathematical Institute,

16 Chambers Street, Edinburgh, 1. 